\title{
Gender Disparities in Alcohol Use Disorder Treatment Among Privately Insured Patients with Alcohol-Associated Cirrhosis
}

\author{
Jessica L. Mellinger (iD, Anne Fernandez, Kerby Shedden, G. Scott Winder, \\ Robert J. Fontana, Michael L. Volk, Frederic C. Blow, and Anna S. F. Lok
}

\begin{abstract}
Background: The burden of alcohol-associated cirrhosis (AC) is high, and though alcohol cessation improves mortality, many patients fail to engage in alcohol use disorder (AUD) treatment and continue drinking. Our aim was to determine rates, predictors, and outcomes of AUD treatment utilization in AC patients with private insurance.

Methods: We collected data from persons with AC (diagnosed by ICD-9/ICD-10 codes), aged 18 to 64 years, enrolled in the Truven MarketScan Commercial Claims and Encounters database (2009 to 2016). We determined rates and predictors of substance abuse treatment visits as well as rates of alcohol relapse prevention medication prescriptions, weighted to the national employer-sponsored insured population. Effects of AUD treatment utilization on decompensation rates were calculated using proportional hazards regression with propensity score adjustment.

Results: A total of 66,053 AC patients were identified, 32\% were female, and mean age at diagnosis was 54.5 years. About $72 \%$ had insurance coverage for substance abuse treatment. Overall, AUD treatment utilization rates were low, with only $10 \%$ receiving a face-to-face mental health or substance abuse visit and only $0.8 \%$ receiving a Food and Drug Administration (FDA)-approved relapse prevention medication within 1 year of index diagnosis. Women were less likely to receive a face-to-face visit (hazard ratio [HR] $0.84, p<0.001)$ or an FDA-approved relapse prevention medication $(0.89$, $p=0.05$ ) than men. AC patients who had a clinic visit for AUD treatment or used FDA-approved relapse medication showed decreased risk of decompensation at 1 year (HR $0.85, p<0.001$ for either).

Conclusions: AUD treatment utilization is associated with lower decompensation rates among privately insured patients with AC. Women were less likely to utilize AUD treatment visits. Efforts to reduce gender-specific barriers to treatment are urgently needed to improve outcomes.
\end{abstract}

Key Words: Alcoholic Cirrhosis, Utilization, Substance Use Treatment, Female.

A LCHOL-ASSOCIATED liver disease (ALD) is a spectrum of liver damage caused by heavy alcohol use over time, ranging from bland hepatic steatosis to more severe forms of liver damage, such as acute alcoholic hepatitis $(\mathrm{AH})$ and alcohol-associated cirrhosis (AC) (O'Shea et al., 2010; Schwartz and Reinus, 2012). The healthcare and economic burden of ALD is high, both in the United States and worldwide, where AC is estimated to be responsible for nearly half the liver-related deaths (Mellinger et al., 2018a; Rehm et al.,

From the Division of Gastroenterology and Hepatology (JLM, RJF, ASFL), University of Michigan, Ann Arbor, Michigan; Department of Psychiatry (AF, GSW, FCB), University of Michigan, Ann Arbor, Michigan; Department of Statistics (KS), University of Michigan, Ann Arbor, Michigan; Transplantation Institute (MLV), Loma Linda University Health, Loma Linda, California; and VA Center for Clinical Management Research (FCB), Ann Arbor, Michigan.

Received for publication July 31, 2018; accepted November 26, 2018.

Reprint requests: Jessica L. Mellinger, MD MSc, 1500 E. Medical Center Dr., 3912 Taubman Center, SPC 5362, Ann Arbor, MI 48109; Tel.: 734-936-8745; Fax: 734-763-4574; E-mail: jmelling@med.umich.edu

(C) 2019 by the Research Society on Alcoholism.

DOI: 10.1111/acer.13944
2013). In the United States, AC comprises over half the total costs of cirrhosis among the privately insured and is a major reason for liver transplantation (Goldberg et al., 2017; Mellinger et al., 2018a; O'Shea et al., 2010). Ongoing alcohol use is the strongest predictor of mortality in ALD patients, particularly those with AH (Louvet et al., 2017). Between 2002 and 2013, the prevalence of past 12-month alcohol use disorder (AUD) in the United States increased by $49.4 \%$ (Grant et al., 2017). Alcohol use is also increasing worldwide, and in the United States, alcohol-related mortality is increasing (Dwyer-Lindgren et al., 2018; World Health Organization, 2014). Given that AC develops only after years of heavy alcohol use, these increases may result in rising AC morbidity and mortality (Guirguis et al., 2015). Alcohol cessation is the primary intervention, which arrests and, in some cases, reverses ALD (Thiele et al., 2018). Yet utilization and effectiveness of AUD treatment in this population are poorly described.

Behavioral and medication-related therapies for AUD are well studied in the general population, with moderate effect sizes demonstrated in multiple trials, though little evidence to suggest one treatment modality is superior to another across all population categories (Donoghue et al., 2015; Jonas 
et al., 2014; Klimas et al., 2014). For example, in the general population, the number needed to treat to prevent a return to any drinking for acamprosate was 12 and for oral naltrexone was 20 (Jonas et al., 2014). Despite documented benefits, access to AUD treatment in the general population is low, with recent large-scale epidemiologic surveys showing a treatment utilization rate of $7.7 \%$ for past year AUD, and $19.8 \%$ for lifetime AUD (Hasin and Grant, 2015). Individuals with AUD that do seek treatment tend to be older ( $\geq 30$ years old), are more likely to be men, report more severe and longer histories of substance use problems, and have co-occurring mood disorders (Khan et al., 2013). There are various reasons individuals with AUD forgo treatment (Greenfield et al., 2007; Khan et al., 2013). Structural barriers such as lack of insurance coverage are experienced by both men and women (Verissimo and Grella, 2017). Women, however, report more attitudinal barriers, more perceived stigma and financial problems, and conflicting family and child care responsibilities (Green et al., 2009; Khan et al., 2013; Verissimo and Grella, 2017).

Utilization of AUD treatment in patients with ALD has been less well evaluated. A recent systematic review of psychosocial, abstinence-focused treatments for AUDs among patients with chronic liver disease found only 5 randomized controlled trials in the literature, 3 of which included AC patients exclusively (Khan et al., 2016; Kuchipudi et al., 1990; Weinrieb et al., 2011; Willenbring and Olson, 1999). Only 1 randomized controlled trial of combined motivational enhancement therapy and cognitive behavioral therapy embedded in the medical clinic showed significantly higher rates of abstinence in the treatment group compared to controls (Willenbring and Olson, 1999). In terms of medicationbased treatments, research in AC patients is even more scarce, with only a single randomized trial of baclofen, an off-label relapse prevention medication, demonstrating a 2 -fold increase in abstinence among $42 \mathrm{AC}$ patients receiving baclofen for 12 weeks versus placebo (Addolorato et al., 2007).

Given the clearly established benefits of alcohol cessation in AC patients and the dearth of research on treatment utilization, the aims of our study were to: (i) assess the rates and predictors of AUD treatment utilization among adults with AC in a large, nationally representative data set of privately insured Americans; and (ii) determine whether utilization of AUD treatment is associated with improved clinical outcomes.

\section{MATERIALS AND METHODS}

\section{Population}

The cohort of AC patients was drawn from the Truven Analytics MarketScan Commercial Claims and Encounters database from 2009 to 2016. In the United States, private insurance accounts for approximately $50 \%$ of the total insurance market, with the overwhelming majority of privately insured individuals receiving coverage via their employer. MarketScan compiles claims from $>100$ million employed persons and their dependents across all regions of the United States, representing well over $50 \%$ of the employer- sponsored insurance market. MarketScan data include claims for enrollees across inpatient, outpatient, facility, and pharmaceutical claims and have been used in multiple large-scale medical and surgical studies of healthcare delivery and costs (Mellinger et al., 2018a).

\section{Inclusion Criteria and Alcoholic Cirrhosis Case Ascertainment}

The initial data set included all patients from 2009 to 2016, aged 18 to 64 years, who had at least a single ICD-9 or ICD-10 code for cirrhosis and at least 1 year of continuous enrollment, including the index cirrhosis diagnosis date (see the Supplementary Appendix). Age was capped at 64 given that most patients aged 65 years and older transition onto Medicare and are thus lost from the data set. All data were restricted to the continuous enrollment period containing the cirrhosis diagnosis. AC was defined using previously published criteria as a single ICD-9 or ICD-10 code for alcohol-AC or a code for cirrhosis without mention of alcohol plus a code for alcohol use or an alcohol-related comorbidity (see the Supplementary Appendix) (Beste et al., 2015; Mellinger et al., 2018a). Patients with both $\mathrm{AC}$ and hepatitis $\mathrm{C}$ (HCV) codes were counted as having AC. Cirrhosis-related complications, such as ascites and hepatic encephalopathy, were defined by ICD-9 or ICD-10 codes (see the Supplementary Appendix). Decompensated AC was defined as an AC diagnosis code and a diagnosis code for a portal hypertensive complication (ascites, hepatic encephalopathy, or variceal bleeding). Single diagnosis codes for cirrhosis and for portal hypertensive complications have been validated in administrative data and found to have positive predictive values of $80 \%$ or greater (Kramer et al., 2008; Nehra et al., 2013). Medical comorbidities were estimated using the Elixhauser comorbidity scale with the liver disease and alcohol abuse categories excluded as these were accounted for separately in our models (Elixhauser et al., 1998). An indicator variable for whether or not the enrollee had insurance coverage for mental health and substance abuse (MHSA) care was included. Census regions were used as geographic variables for predictors of AUD treatment access with census region 3 (South) as the reference region.

\section{MHSA Treatment Ascertainment}

We defined comorbid depression or anxiety by ICD-9 or ICD-10 code. Because substance use is frequently assessed and treated alongside other mental health issues and is often comorbid with alcohol use and misuse, alcohol use treatment was defined as either a substance use or mental health outpatient visit or a prescription for a U.S. Food and Drug Administration (FDA)-approved alcohol relapse prevention medication. We performed additional analyses of non-FDA-approved alcohol relapse prevention medications. An MHSA treatment visit was defined as a Current Procedural Terminology (CPT) code for a face-to-face outpatient visit combined with a claims-based service category code, indicating a substance abuserelated claim was submitted on the same day, or a CPT code for a face-to-face outpatient visit with a provider code, indicating that the visit was conducted by a psychiatrist or psychologist. FDAapproved (disulfiram, naltrexone, and acamprosate) and nonFDA-approved (baclofen, gabapentin, and topiramate) medications for alcohol relapse prevention were identified in the pharmaceutical claims data set. Enrollees were required to have a 90-day or greater continuous prescription in order to eliminate short courses of treatment, which would be expected to have less influence on alcohol use. Analyses of medication prescriptions were restricted to enrollees with prescription drug coverage.

\section{Statistical Methods}

We calculated baseline proportions of covariates present at index diagnosis. $\mathrm{AC}, \mathrm{HCV}$, and diabetes were treated as time 
invariant, meaning that if a diagnosis appeared at any time in the enrollment period, enrollees were counted as having these diagnoses. We calculated pre- and postindex cirrhosis diagnosis rates of MHSA treatment utilization. Rates for medication use (antidepressants, alcohol relapse prevention medications) were calculated in the population of patients with prescription drug coverage, while rates of MHSA clinic visits were calculated in the total population with or without prescription drug coverage. We modeled the cumulative event rates for major events of interest (prescriptions for FDA or non-FDA-approved relapse prevention medication and MHSA outpatient visits) at 1 month, 1 , and 2 years postindex diagnosis and weighted these to reflect the national population with private, employer-sponsored insurance as previously reported (Mellinger et al., 2018a). To identify predictors of treatment utilization, we used proportional hazards regression with propensity score adjustment based on multiple covariates and 100 strata to model the hazard from the index AC diagnosis to the first occurrence of a given form of alcohol use treatment (visit or medication). Models were fit using standard methods for proportional hazards estimation in the setting of time-varying covariates. Patients who received liver transplant were censored at time of transplant. For additional analyses of non-FDA-approved relapse prevention medications, diabetes was considered an independent predictor given the high occurrence of gabapentin use for diabetic neuropathy.

\section{Clinical Outcomes}

Our primary clinical outcome was hepatic decompensation defined as ascites, hepatic encephalopathy, or variceal bleeding. We assessed the association between MHSA treatment and decompensation using proportional hazards regression with time-varying covariates as in the above methods for predictors of treatment access. Patients with decompensation diagnoses at index diagnosis were excluded from the model. Propensity score adjustment based on multiple covariates and 100 strata were used as in the access model above. The effects of MHSA treatment on decompensation were assessed in 3 separate models: (i) FDA-approved medications alone, (ii) MHSA treatment visits alone, and (iii) FDA-approved medications and MHSA treatment visits as a composite variable.

\section{RESULTS}

\section{Patient Characteristics}

A total of 66,053 patients with AC were identified, approximately one-third were female (see Table 1). Mean age at diagnosis was 53.5 years. Of these, $72 \%$ had MHSA coverage under their insurance plans and 87\% had prescription drug coverage. Roughly one-quarter (28\%) also had HCV, and 53\% were decompensated. Depression and anxiety were present in 16 and $12 \%$, respectively, at index cirrhosis diagnosis. Baseline FDAapproved alcohol relapse prevention medication prescriptions were rare $(0.4 \%)$, while non-FDA-approved relapse medications were more common (3.2\%). Gabapentin made up the greatest proportion of non-FDA-approved relapse prevention medication prescriptions at index cirrhosis diagnosis $(2.6 \%)$ (see Fig. 1).
Table 1. Population Characteristics at Index Alcoholic Cirrhosis Diagnosis

\begin{tabular}{|c|c|}
\hline Characteristics & $\begin{array}{c}\text { Total AC patients } \\
n=66,053 \\
N(\%)\end{array}$ \\
\hline Female & $21,442(32 \%)$ \\
\hline Mean age (years) & 53.5 \\
\hline $\begin{array}{l}\text { Mental health/substance abuse } \\
\text { treatment coverage }\end{array}$ & $47,505(72 \%)$ \\
\hline Prescription drug coverage & $57,632(87 \%)$ \\
\hline Mean Elixhauser & 3.53 \\
\hline Hepatitis C & $18,817(28 \%)$ \\
\hline Decompensation $^{\mathrm{a}}$ & $35,069(53 \%)$ \\
\hline Anxiety & $7,642(12 \%)$ \\
\hline Depression & $10,652(16 \%)$ \\
\hline $\begin{array}{l}\text { Any FDA-approved alcohol relapse } \\
\text { prevention medication }\end{array}$ & $275(0.4 \%)$ \\
\hline Acamprosate & $122(0.2 \%)$ \\
\hline Disulfiram & $133(0.2 \%)$ \\
\hline Naltrexone & $99(0.1 \%)$ \\
\hline
\end{tabular}

adDecompensation defined as presence of ascites, variceal bleeding, or hepatic encephalopathy.

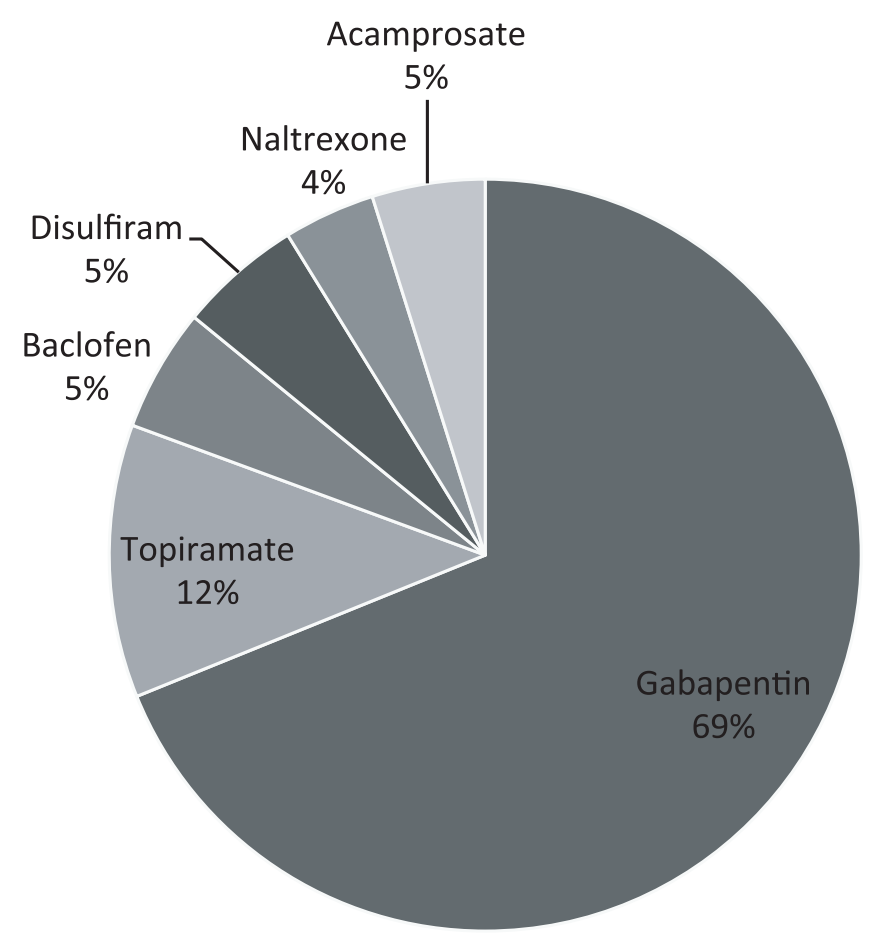

Fig. 1. Proportion of alcohol relapse prevention medication prescriptions at index diagnosis, by type of prescription.

\section{Rates of AUD Treatment and Alcohol Relapse Prevention Utilization After Index AC Diagnosis in the National Employer-Sponsored Insurance Population}

FDA-approved alcohol relapse prevention medication use and MHSA visits increased over time, from $0.2 \%$ for medications and $3.0 \%$ for MHSA visits at 1 month after AC diagnosis to 0.8 and $10.1 \%$, respectively, at 1 year, and to 1.2 and $14.5 \%$, respectively, at 2 years (see Fig. 2). MHSA visits alone accounted for most of the AUD treatment 
utilization. Rates of FDA-approved alcohol relapse prevention medications alone were low, increasing from $0.2 \%$ at 1 month to $1.2 \%$ at 2 years, with similar rates of acamprosate and naltrexone usage and a lower rate of usage of disulfiram. Use of non-FDA-approved medications that have been suggested to prevent alcohol relapse was more common and dominated by gabapentin use $(8.3 \%$ at 2 years postdiagnosis).

\section{Predictors of AUD Treatment Utilization in the MarketScan Population}

Women were less likely to utilize face-to-face MHSA clinic visits (hazard ratio [HR] 0.84, $p<0.001$ ) and FDAapproved alcohol relapse prevention medications (HR $0.89, p=0.05$ ) (see Fig. 3). In analyses of separate outcomes (MHSA visits or FDA-approved relapse prevention medications), patients with MHSA insurance coverage were more likely to utilize MHSA clinic visits (HR 1.32, $p<0.001)$ and FDA-approved alcohol relapse prevention medications (HR 1.88, $p<0.001$ ) than those who did not. Older patients and those with decompensation were less likely to have attended a face-to-face MHSA clinic visit (HR 0.97 and HR 0.89, $p<0.001$ for both) while patients with depression and anxiety diagnoses were much more likely to have such visits (HR 2.17 and HR 1.47, $p<0.001$ for both). See Table 2 for findings from the composite outcome of either MHSA visit or FDAapproved relapse prevention medication.
Within the cohort of AC patients with prescription drug coverage, women were less likely to receive FDA-approved alcohol relapse prevention medication prescriptions (HR $0.89, p=0.05$ ) than men and those with MHSA insurance coverage were more likely to receive these prescriptions (HR $1.88, p<0.001)$ than those without MHSA insurance coverage. Having a depression diagnosis was the strongest predictor of FDA-approved alcohol relapse prevention medication utilization (HR 3.62, $p<0.001$ ) as was having an anxiety diagnoses though the effect was attenuated compared to depression (HR 1.32, $p<0.001$ ). In additional analyses examining utilization of non-FDA-approved relapse prevention medications, women were more likely to receive these prescriptions as well even after discounting gabapentin use (any non-FDA medication: HR 1.33, $p<0.001$; topiramate and baclofen only (no gabapentin): HR 1.84, $p<0.001$ ).

\section{Clinical Outcome: Hepatic Decompensation in the MarketScan Population}

Approximately one-fifth (19\%) of patients had new diagnosis codes for hepatic decompensation within 1 year after index diagnosis of AC. In multivariate models, utilization of an MHSA clinic visit alone had a protective effect on risk of decompensation (HR 0.89, $p<0.001$ ). Although the number was small, use of FDA-approved alcohol relapse prevention medication alone had a greater effect on decreasing the risk of decompensation (HR 0.65, $p<0.001$ ). In a composite model analyzing effects of having either an MHSA visit or

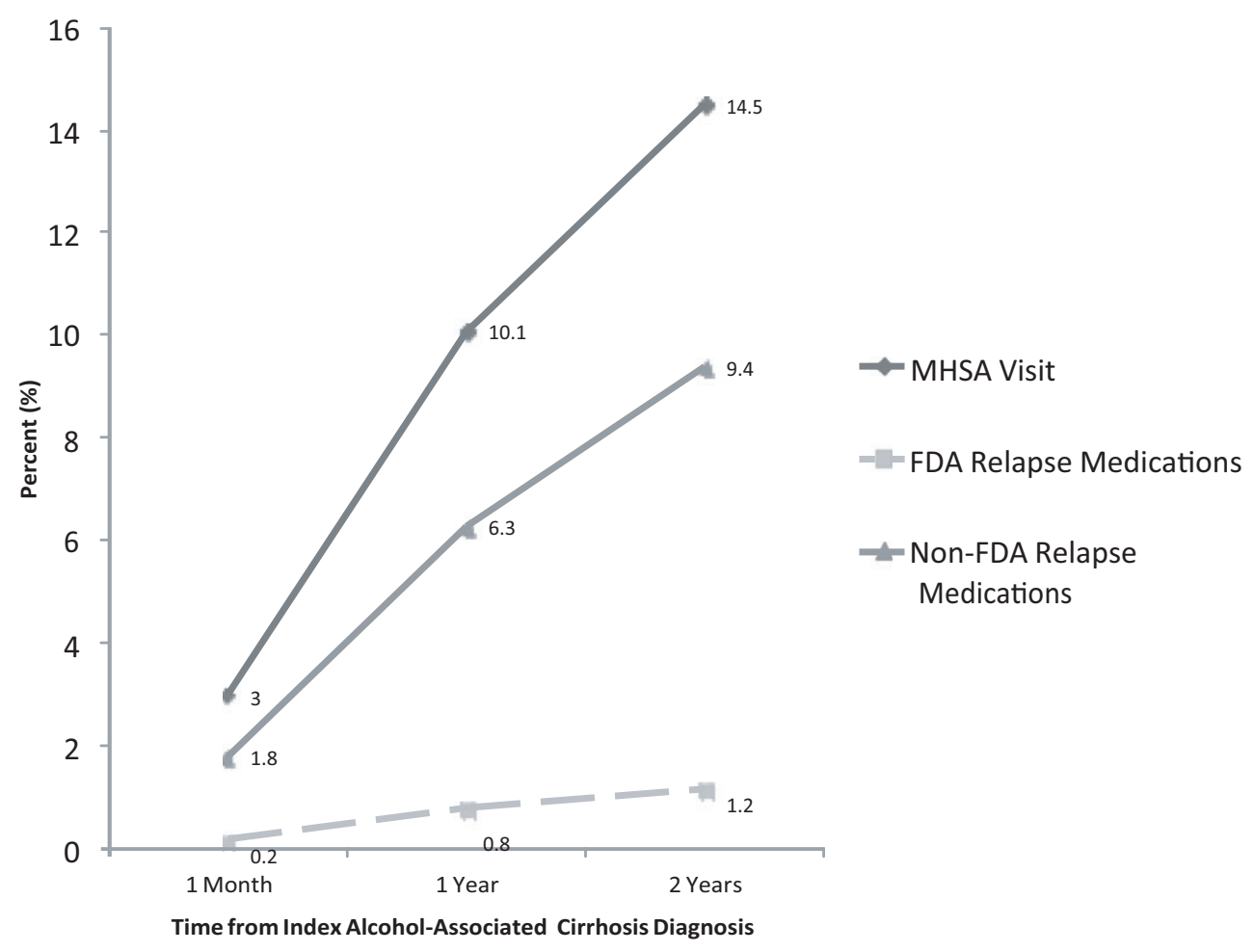

Fig. 2. Rates of MHSA treatment utilization at 1 month, 1 , and 2 years postindex diagnosis. 


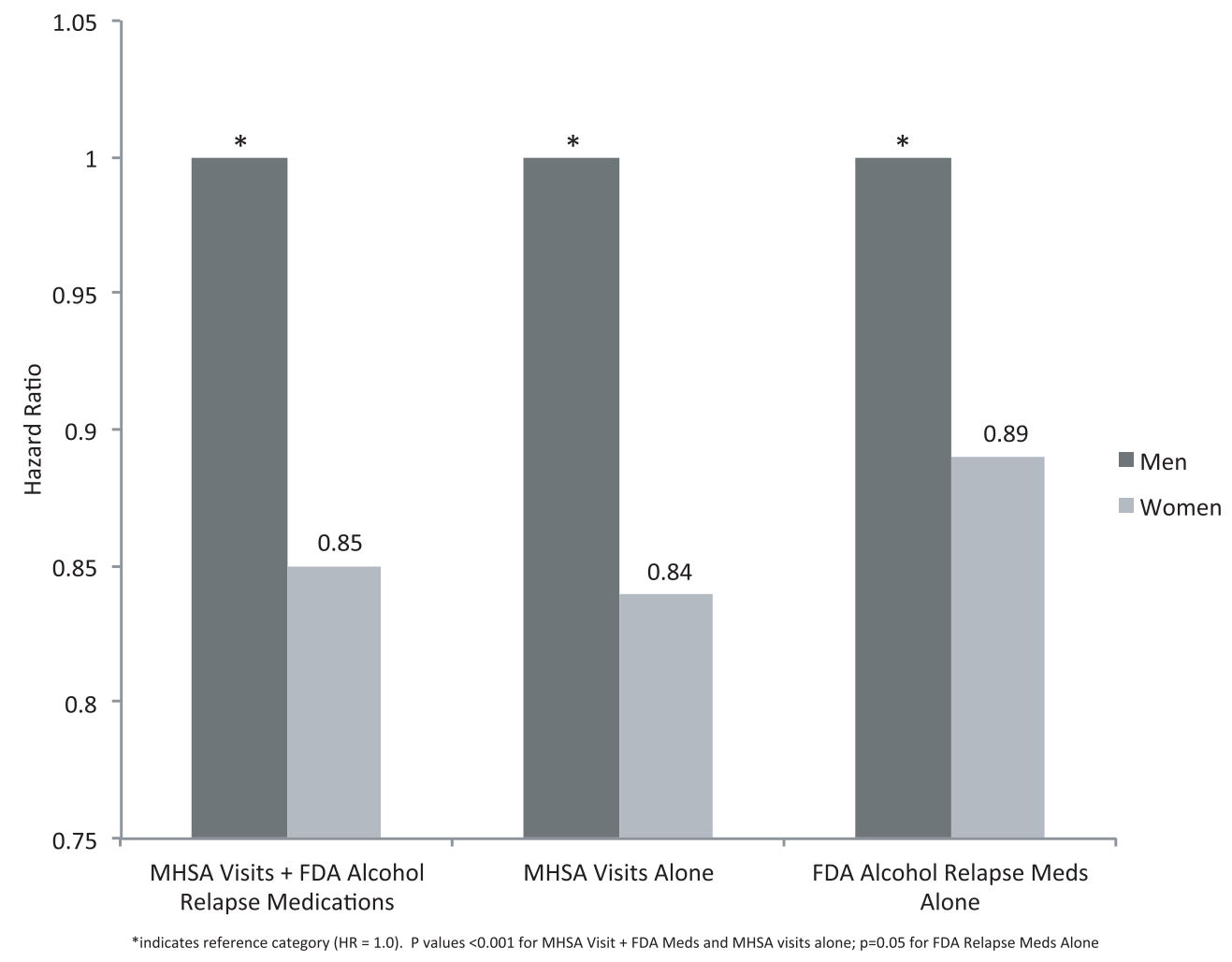

Fig. 3. Gender differences in MHSA treatment utilization.

Table 2. Predictors of Access to MHSA Visits or FDA Alcohol Relapse Prevention Medications

\begin{tabular}{|c|c|c|}
\hline Variable & $\begin{array}{c}\text { Hazard ratio } \\
\text { (95\% confidence interval) }\end{array}$ & $p$-Value \\
\hline MHSA insurance coverage & 1.34 (1.28 to 1.40$)$ & $<0.001$ \\
\hline Female & $0.85(0.82$ to 0.88$)$ & $<0.001$ \\
\hline Decompensated cirrhosis & 0.89 (0.86 to 0.92$)$ & $<0.001$ \\
\hline Diabetes & 0.79 (0.76 to 0.82$)$ & $<0.001$ \\
\hline Age at diagnosis & $0.97(0.97$ to 0.98$)$ & $<0.001$ \\
\hline Hepatitis C & $1.00(0.97$ to 1.04$)$ & 0.70 \\
\hline Anxiety & 1.47 (1.41 to 1.53$)$ & $<0.001$ \\
\hline Depression & 2.21 (2.13 to 2.30$)$ & $<0.001$ \\
\hline Elixhauser & 1.41 (1.37 to 1.44$)$ & $<0.001$ \\
\hline PCP visit & 1.60 (1.54 to 1.67$)$ & $<0.001$ \\
\hline GI clinic visit & 1.07 (1.03 to 1.11$)$ & $<0.001$ \\
\hline South $^{\mathrm{a}}$ & 1.00 & a \\
\hline Northeast & 1.33 (1.27 to 1.39$)$ & $<0.001$ \\
\hline Midwest & $1.20(1.15$ to 1.26$)$ & $<0.001$ \\
\hline West & $1.53(1.46$ to 1.60$)$ & $<0.001$ \\
\hline
\end{tabular}

andicates reference category census region.

an FDA-approved relapse medication, reduction in risk of decompensation was similar to that of MHSA visit alone likely due to infrequent use of FDA-approved relapse medication (HR 0.85, $p<0.001$ ) (see Table 3 ). Older age was associated with a minimally lower risk of decompensation (HR 0.998, $p<0.001$ ). There was a gender and Elixhauser interaction, resulting in slightly lower risk of decompensation (HR 0.96, $p<0.001$ ). Diagnoses of depression and use of antidepressant medications were both associated with lower
Table 3. Substance Abuse Treatment Utilization Effects on Occurrence of Hepatic Decompensation Within 1 Year Following Index Cirrhosis Diagnosis

\begin{tabular}{llc}
\hline Variable & $\begin{array}{c}\text { HR }(95 \% \text { confidence } \\
\text { interval })\end{array}$ & $p$-Value \\
\hline $\begin{array}{l}\text { Composite MHSA visit } \\
\text { and/or FDA medication }\end{array}$ & $0.85(0.82$ to 0.87$)$ & $<0.001$ \\
$\begin{array}{ll}\text { Female: Elixhauser } \\
\text { Diabetes }\end{array}$ & $0.96(0.94$ to 0.97$)$ & $<0.001$ \\
Age at diagnosis & $0.98(0.96$ to 0.99$)$ & $<0.001$ \\
HCV & $0.998(0.998$ to 0.999$)$ & 0.02 \\
Hepatorenal syndrome & $1.22(1.20$ to 1.24$)$ & $<0.001$ \\
Acute kidney injury & $2.63(2.51$ to 2.76$)$ & $<0.001$ \\
Infection & $1.37(1.34$ to 1.40$)$ & $<0.001$ \\
Depression & $1.16(1.14$ to 1.18$)$ & $<0.001$ \\
Antidepressant & $0.77(0.76$ to 0.79$)$ & $<0.001$ \\
medication prescription & $0.99(0.97$ to 1.01$)$ & $<0.001$ \\
PCP visit & & $<0.001$ \\
Gl visit & $0.83(0.82$ to 0.84$)$ & $<0.001$ \\
South & $1.36(1.35$ to 1.38$)$ & \\
Northeast & 1.00 & $<0.001$ \\
Midwest & $0.95(0.93$ to 0.97$)$ & 0.002 \\
West & $1.03(1.01$ to 1.05$)$ & $<0.001$ \\
\hline
\end{tabular}

anteraction between gender and Elixhauser score.

bIndicates reference category for geographic comparisons.

rates of hepatic decompensation (HR 0.77 and HR 0.99, $p<0.001$ for both). In both models of MHSA visits and FDA medications, primary care visits were associated with decreased risk of decompensation (HR 0.82 and 0.83 , $p<0.001$ for both). 


\section{DISCUSSION}

In this large study of privately insured patients with $\mathrm{AC}$, overall utilization of AUD treatment was low, despite a high rate of MHSA insurance coverage. Gender influenced utilization, with women being less likely to obtain both a face-to-face MHSA clinic visit and FDA-approved relapse prevention medications. Those who utilized alcohol use treatment, whether men or women, were significantly less likely to decompensate at 1 year after index diagnosis compared to those who did not utilize such treatment, even when adjusting for comorbidities, such as HCV and diabetes. In a best-case scenario, men and women who access AUD treatment early, whether relapse prevention medications or MHSA clinic visits may avoid progression to AC and decompensation with its subsequent high mortality and possible need for transplant. Those who fail to access AUD treatment and continue drinking may go on to further decompensate, bringing with it higher mortality, more inpatient hospital admissions, higher costs, and potential need for transplant. For those that do not access formal AUD treatment, transplant may be denied given the requirement for alcohol cessation and formal AUD treatment at most U.S. liver transplant centers.

Similar to reported low rates of MHSA utilization in the general population, only $10 \%$ of privately insured AC patients utilized an MHSA clinic visit within 1 year after their index diagnosis. While lack of insurance coverage is frequently cited as a major reason for lack of utilization of AUD treatment, our study population had private insurance with a high rate of MHSA coverage, and MHSA coverage was weakly correlated with utilization. This fits with existing data, showing that barriers to AUD treatment are related more to patient attitudes than to structural insurance issues (Khan et al., 2013; Verissimo and Grella, 2017). The gender imbalance in alcohol treatment utilization in the privately insured AC population is consistent with research in the broader AUD population, indicating women are less likely to receive inpatient, outpatient, emergency room, or other face-to-face treatment for AUD relative to men and are less likely to attend specialty addiction treatment services (Khan et al., 2013). In a mixed-methods study of AC patients, men and women identified attitudinal barriers as major causes for lack of AUD treatment uptake (Mellinger et al., 2018b).

Barriers to AUD treatment differ between men and women with women experiencing more barriers relative to men, including higher perceived stigma and other "attitudinal" barriers as well as higher economic and time barriers (Green et al., 2009; Khan et al., 2013; Verissimo and Grella, 2017). Diagnosis of AUDs in women is challenging as alcohol screening tools have lower specificity among women, and opportunities for diagnosis are frequently missed (Bradley et al., 1998; Volk et al., 1997). For example, among AC patients in the United Kingdom, women were less likely to have alcohol use recorded and were overall less likely to interact with the healthcare system in the period prior to their AC diagnosis (Otete et al., 2015). Despite evidence that women are less likely to seek face-to-face treatment, when women do access psychosocial treatment, they may have better treatment outcomes than men (Green et al., 2009; Sugarman et al., 2017). Low treatment utilization among women is particularly concerning given a higher proportion of American women now meet criteria for AUD than ever before, with rates rising more rapidly in women than in men (Grant et al., 2017). In the liver disease population, women frequently develop $\mathrm{AC}$ and $\mathrm{AH}$ at lower amounts of alcohol consumed and shorter time frames compared to men, suggesting that rates of ALD, as demonstrated in our earlier study, will rise more rapidly in women, increasing the urgency for connection to professional AUD treatment (Mellinger et al., 2018a; Nielsen et al., 2017; Szabo, 2018).

We found that only a very small fraction $(0.8 \%)$ of AC patients received an FDA-approved relapse prevention medication despite having insurance coverage for health care and prescriptions. It is possible that this underutilization of AUD treatment is related to concerns about liver toxicity for naltrexone and disulfiram. Acamprosate, which is not metabolized by the liver, has no reported instances of clinically significant hepatotoxicity and may be safe, but it has not been explicitly tested in those with advanced liver disease. Only baclofen, a nonFDA-approved relapse prevention medication, has been tested in a small randomized trial in patients with AC, showing a benefit at 12 weeks of treatment with baclofen $10 \mathrm{mg} 3$ times daily with an acceptable side effect profile (Addolorato et al., 2007). In our study, baclofen use, like other relapse prevention medications, was low.

We found that a 90-day prescription for an FDAapproved alcohol relapse prevention medication was associated with an even greater reduction in decompensation compared to an MHSA clinic visit. While our findings do not demonstrate causation, given alcohol's deleterious effects on cirrhosis and portal hypertensive complications, one possible mechanism for their positive influence may be via improved rates of alcohol abstinence, similar to the mechanism for MHSA visit effect (Lucey et al., 2008). Our additional analyses indicated that non-FDAapproved relapse prevention medications also had a large effect on decompensation rates. Gabapentin, a neuromodulator typically prescribed for seizures or peripheral neuropathy, has an increasing literature base supporting its effects on relapse prevention, primarily through modulation of alcohol craving, and was the most common relapse prevention medication prescribed, though it is likely much more frequently prescribed for other indications, such as neuropathy (Mason et al., 2014). Similarly, topiramate has some evidence, though with smaller studies, for reducing alcohol use (Johnson et al., 2003, 2007). While the indication for use of gabapentin, topiramate, and baclofen in our patient population cannot be determined, the associated beneficial effect on decompensation rates is worthy of further study. 
Diagnoses of depression and antidepressant medication use were associated with increased utilization of MHSA care (both visits and FDA-approved relapse medications) and lower rates of decompensation. This effect may be related to the fact that patients diagnosed with depression or anxiety and prescribed antidepressant medications to treat these conditions are connected to the medical system and likely utilize care overall to a greater degree. Comorbid mental illness is common in AUD patients and associated with increased, though inconsistent, utilization of primary medical care (Ford et al., 2005). The complex psychosocial milieu and medical comorbidities AC patients confront, combined with complex medication prescribing in advanced liver disease, warrant the establishment of integrated clinics with colocated substance use professionals working alongside hepatologists to appropriately care for these complex patients.

There were several limitations to our study. First, this database only assesses those with private insurance and a claim for healthcare services and may thus lack generalizability to nonprivately insured populations. Second, alternate supports for sobriety in the community, such as mutual aid societies like Alcoholics Anonymous, would not be reflected in our data and are difficult to measure. Third, behavioral interventions for alcohol treatment, such as psychoeducation or other brief interventions, are frequently delivered by nonaddiction specialists and may have occurred outside MHSA visits. We attempted to mitigate this limitation by broadening our ascertainment to include both visits conducted by a psychiatrist or psychologist and visits where a service category code related to substance abuse treatment was entered, regardless of the specialty of the medical provider entering the code. Fourth, we are unable to determine the content of the substance abuse intervention and cannot ascertain the effectiveness of different modalities of alcohol use treatment. Fifth, AUD treatment efficacy may be influenced by endogenous factors influencing a patient's motivation to take up alcohol treatment such that patients who engage in treatment are more likely to stop drinking for reasons other than the treatment effectiveness. Sixth, the requirement for 1 year of continuous enrollment may have biased the population toward a healthier population by excluding those who died within 1 year of diagnosis or lost insurance due to disability.

In conclusion, patients with AC utilize AUD treatment at low rates within 1 year after AC diagnosis, with women less likely to receive AUD treatment compared to men. Alcohol cessation is the only intervention known to improve mortality in patients with ALD, and receipt of AUD treatment in our study was significantly associated with improved outcomes. As such, efforts to improve utilization rates of MHSA care, including treatment of comorbid mental health conditions, are necessary. Integrated care combining professional mental health and hepatology care and the development of novel behavioral treatments for this population are urgently needed.

\section{FUNDING}

This work was supported by an American Association for the Study of Liver Diseases 2016 Clinical and Translational Research Award.

\section{CONFLICT OF INTEREST}

The authors have no relevant conflicts of interest to report.

\section{AUTHOR CONTRIBUTIONS}

JLM: Study design, analysis and interpretation of data, figure creation, manuscript drafting, and revision; AF: Analysis and interpretation of data, manuscript drafting, and revision; KS: Study design, statistical analysis and interpretation of data, and manuscript revision; GSW: Manuscript revision; RJF: Manuscript revision; MLV: Interpretation of data and manuscript revision; FCB: Interpretation of data and manuscript revision; ASFL: Interpretation of data and manuscript revision.

\section{REFERENCES}

Addolorato G, Leggio L, Ferrulli A, Cardone S, Vonghia L, Mirijello A, Abenavoli L, D'Angelo C, Caputo F, Zambon A, Haber PS, Gasbarrini G (2007) Effectiveness and safety of baclofen for maintenance of alcohol abstinence in alcohol-dependent patients with liver cirrhosis: randomised, double-blind controlled study. Lancet 370:1915-1922.

Beste LA, Leipertz SL, Green PK, Dominitz JA, Ross D, Ioannou GN (2015) Trends in burden of cirrhosis and hepatocellular carcinoma by underlying liver disease in US veterans, 2001-2013. Gastroenterology 149:1471-1482.

Bradley KA, Boyd-Wickizer J, Powell SH, Burman ML (1998) Alcohol screening questionnaires in women: a critical review. J Am Med Assoc 280:166-171.

Donoghue K, Elzerbi C, Saunders R, Whittington C, Pilling S, Drummond $C$ (2015) The efficacy of acamprosate and naltrexone in the treatment of alcohol dependence, Europe versus the rest of the world: a meta-analysis. Addiction 110:920-930.

Dwyer-Lindgren L, Bertozzi-Villa A, Stubbs RW, Morozoff C, Shirude S, Unützer J, Naghavi M, Mokdad AH, Murray CJL (2018) Trends and patterns of geographic variation in mortality from substance use disorders and intentional injuries among US counties, 1980-2014. J Am Med Assoc 319:1013-1023.

Elixhauser A, Steiner C, Harris DR, Coffey RM (1998) Comorbidity measures for use with administrative data. Med Care 36:8-27.

Ford JD, Trestman RL, Tennen H, Allen S (2005) Relationship of anxiety, depression and alcohol use disorders to persistent high utilization and potentially problematic under-utilization of primary medical care. Soc Sci Med 61:1618-1625.

Goldberg D, Ditah IC, Saeian K, Lalehzari M, Aronsohn A, Gorospe EC, Charlton M (2017) Changes in the prevalence of hepatitis C virus infection, nonalcoholic steatohepatitis, and alcoholic liver disease among patients with cirrhosis or liver failure on the waitlist for liver transplantation. Gastroenterology 152:1090-1099.

Grant BF, Chou SP, Saha TD, Pickering RP, Kerridge BT, Ruan WJ, Huang B, Jung J, Zhang H, Fan A, Hasin DS (2017) Prevalence of 12month alcohol use, high-risk drinking, and DSM-IV alcohol use disorder in the United States, 2001-2002 to 2012-2013: results from the National 
Epidemiologic Survey on Alcohol and Related Conditions. J Am Med Assoc Psychiatry 74:911-923.

Green BL, Rockhill A, Furrer C (2009) Understanding patterns of substance abuse treatment for women involved with child welfare: the influence of the Adoption and Safe Families Act (ASFA). Am J Drug Alcohol Abuse 32:149-176.

Greenfield SF, Brooks AJ, Gordon SM, Green CA, Kropp F, McHugh RK, Lincoln M, Hien D, Miele GM (2007) Substance abuse treatment entry, retention, and outcome in women: a review of the literature. Drug Alcohol Depend 86:1-21.

Guirguis J, Chhatwal J, Dasarathy J, Rivas J, McMichael D, Nagy LE, McCullough AJ, Dasarathy S (2015) Clinical impact of alcohol-related cirrhosis in the next decade: estimates based on current epidemiological trends in the United States. Alcohol Clin Exp Res 39:2085-2094.

Hasin DS, Grant BF (2015) The National Epidemiologic Survey on Alcohol and Related Conditions (NESARC) Waves 1 and 2: review and summary of findings. Soc Psychiatry Psychiatr Epidemiol 50:1609-1640.

Johnson BA, Ait-Daoud N, Bowden CL, DiClemente CC, Roache JD, Lawson K, Javors MA, Ma JZ (2003) Oral topiramate for treatment of alcohol dependence: a randomised controlled trial. Lancet 361:1677-1685.

Johnson BA, Rosenthal N, Capece JA, Wiegand F, Mao L, Beyers K, McKay A, Ait-Daoud N, Anton RF, Ciraulo DA, Kranzler HR, Mann K, O'Malley SS, Swift RM (2007) Topiramate for treating alcohol dependence: a randomized controlled trial. J Am Med Assoc 298:1641-1651.

Jonas DE, Amick HR, Feltner C, Bobashev G, Thomas K, Wines R, Kim MM, Shanahan E, Gass CE, Rowe CJ, Garbutt JC (2014) Pharmacotherapy for adults with alcohol use disorders in outpatient settings: a systematic review and meta-analysis. J Am Med Assoc 311:1889-1900.

Khan A, Tansel A, White DL, Kayani WT, Bano S, Lindsay J, El-Serag HB, Kanwal F (2016) Efficacy of psychosocial interventions in inducing and maintaining alcohol abstinence in patients with chronic liver disease: a systematic review. Clin Gastroenterol Hepatol 14:191-202.

Khan S, Okuda M, Hasin DS, Secades-Villa R, Keyes K, Lin KH, Grant B, Blanco C (2013) Gender differences in lifetime alcohol dependence: results from the National Epidemiologic Survey on Alcohol and Related Conditions. Alcohol Clin Exp Res 118:1696-1705.

Klimas J, Tobin H, Field CA, O'Gorman CSM, Glynn LG, Keenan E, Saunders J, Bury G, Dunne C, Cullen W (2014) Psychosocial interventions to reduce alcohol consumption in concurrent problem alcohol and illicit drug users. Cochrane Database Syst Rev 95:CD009269.

Kramer JR, Davila JA, Miller ED, Richardson P, Giordano TP, El-Serag HB (2008) The validity of viral hepatitis and chronic liver disease diagnoses in Veterans Affairs administrative databases. Aliment Pharmacol Ther 27:274-282.

Kuchipudi V, Hobein K, Flickinger A, Iber FL (1990) Failure of a 2-hour motivational intervention to alter recurrent drinking behavior in alcoholics with gastrointestinal disease. J Stud Alcohol 51:356-360.

Louvet A, Labreuche J, Artru F, Bouthors A, Saffers P, Mathurin P (2017) Drivers of short- and long-term mortality in severe alcoholic hepatitis: a complex relationship between alcohol relapse and early improvement in liver function. Hepatology 66:1464-1473.

Lucey MR, Connor JT, Boyer TD, Henderson JM, Rikkers LF (2008) Alcohol consumption by cirrhotic subjects: patterns of use and effects on liver function. Am J Gastroenterol 103:1698-1706.

Mason BJ, Quello S, Goodell V, Shadan F, Kyle M, Begovic A (2014) Gabapentin treatment for alcohol dependence: a randomized clinical trial. J Am Med Assoc Intern Med 174:70-77.

Mellinger JL, Shedden K, Winder GS, Tapper E, Adams M, Fontana RJ, Volk ML, Blow FC, Lok ASF (2018a) The high burden of alcoholic cirrhosis in privately insured persons in the United States. Hepatology 68:872-882.

Mellinger JL, Winder GS, DeJonckheere M, Fontana RJ, Volk ML, Lok ASF, Blow FC (2018b) Misconceptions, preferences and barriers to alcohol use disorder treatment in alcohol-related cirrhosis. J Subst Abuse Treat 91:20-27.

Nehra MS, Ma Y, Clark C, Amarasingham R, Rockey DC, Singal AG (2013) Use of administrative claims data for identifying patients with cirrhosis. J Clin Gastroenterol 47:50-54.

Nielsen JK, Olafsson S, Bergmann OM, Runarsdottir V, Hansdottir I, Sigurdardottir R, Björnsson ES (2017) Lifetime drinking history in patients with alcoholic liver disease and patients with alcohol use disorder without liver disease. Scand J Gastroenterol 52:762-767.

O'Shea RS, Dasarathy S, McCullough AJ; Practice Guideline Committee of the American Association for the Study of Liver Diseases (2010) Alcoholic liver disease. Hepatology 51:307-328.

Otete HE, Orton E, West J, Fleming KM (2015) Sex and age differences in the early identification and treatment of alcohol use: a population-based study of patients with alcoholic cirrhosis. Addiction 110:1932-1940.

Rehm J, Samokhvalov AV, Shield KD (2013) Global burden of alcoholic liver diseases. J Hepatol 59:160-168.

Schwartz JM, Reinus JF (2012) Prevalence and natural history of alcoholic liver disease. Clin Liver Dis 16:659-666.

Sugarman DE, Campbell ANC, Iles BR, Greenfield SF (2017) Technologybased interventions for substance use and comorbid disorders: an examination of the emerging literature. Harvard Rev Psychiatry 25:123-134.

Szabo G (2018) Women and alcoholic liver disease-warning of a silent danger. Nat Rev Gastroenterol Hepatol 12:231-254.

Thiele M, Rausch V, Fluhr G, Kjærgaard M, Piecha F, Mueller J, Straub BK, Lupșor-Platon M, De-Ledinghen V, Seitz HK, Detlefsen S, Madsen B, Krag A, Mueller S (2018) Controlled attenuation parameter and alcoholic hepatic steatosis: diagnostic accuracy and role of alcohol detoxification. J Hepatol 68:1025-1032.

Verissimo ADO, Grella CE (2017) Influence of gender and race/ethnicity on perceived barriers to help-seeking for alcohol or drug problems. J Subst Abuse Treat 75:54-61.

Volk RJ, Steinbauer JR, Cantor SB, Holzer CE (1997) The Alcohol Use Disorders Identification Test (AUDIT) as a screen for at-risk drinking in primary care patients of different racial/ethnic backgrounds. Addiction 92:197-206.

Weinrieb RM, Van Horn DHA, Lynch KG, Lucey MR (2011) A randomized, controlled study of treatment for alcohol dependence in patients awaiting liver transplantation. Liver Transpl 17:539-547.

Willenbring ML, Olson DH (1999) A randomized trial of integrated outpatient treatment for medically ill alcoholic men. Arch Intern Med 159:19461952.

World Health Organization (2014) Global Status Report on Alcohol and Health. World Health Organization, Geneva.

\section{SUPPORTING INFORMATION}

Additional supporting information may be found online in the Supporting Information section at the end of the article.

Appendix. ICD-9 and ICD-10 codes used for cohort selection and comorbidities 